\title{
Sie \\ First report of bacterial soft rot of carrot caused by Klebsiella variicola in India
}

\author{
B.S. Chandrashekar*, M.K. Prasannakumar, M.E. Puneeth, Kalavati Teli, K. Priyanka, H.B. Mahesh and Radhika U. Desai
}

Pathogenomic Laboratory, Department of Plant Pathology, College of Agriculture, Gandhi Krushi Vignana Kendra, University of Agricultural Sciences, Bengaluru, Karnataka, India

*E-mail: chandruchiru.93@gmail.com

Received: 12 Oct 2017. Published: 18 May 2018. Keywords: anaerobic, enrichment host

During 2016-2017, carrots (Daucus carota) with severe soft rot symptoms on the tap root, associated with a foul odour, wilting and collapse of foliage (Fig. 1), were collected from the rural districts of Kolar, Chikkaballapura and Bengaluru of Karnataka state, India. Disease incidence was 20-30\% in affected fields. Samples of two or three roots were collected from each of ten different fields. Isolation was done using green pepper fruits as enrichment hosts (Fig. 2), followed by streaking on nutrient agar as described by Akbar et al. (2015). The resulting bacterial colonies were whitish to dull white, mucoid, raised and slimy on nutrient agar (Fig. 3). Ten isolates, one from each sample were identified as Gram-negative and anaerobic. In biochemical tests performed according to Brenner et al. (2004), all isolates were positive for catalase and nitrate reductase, and used citrate, glucose, lactose, maltose, malonate and celloboise, but were negative for oxidase, indole and acetoin tests. They produced mucoid growth on Luria Bertani medium, grew at $37^{\circ} \mathrm{C}$ and on $5 \% \mathrm{NaCl}$.

To confirm pathogenicity, bacterial suspensions (100 $\mu$, approx. $10^{8} \mathrm{CFU} / \mathrm{ml}$ ) were injected into whole roots and pipetted onto $5 \mathrm{~mm}$ thick carrot slices (Michalik et al., 1992) and incubated at $30^{\circ} \mathrm{C}$ and $90 \%$ relative humidity in a growth chamber. Sterile distilled water was used as a negative control. Water-soaked lesions developed on whole roots after three days, leading to soft rotting with a foul odour after four days (Fig. 4). Brown lesions were observed on carrot slices after three days and the degree of rotting varied between different isolates. Bacteria with identical colony characteristics were re-isolated from all inoculated rotted tissues.

Amplification of the 16S rDNA gene was done using universal primers fD1 (5'-GAGTTTGATCCTGGTCA-3') and rP2 (5'-ACGGCTACCTTGTTACGACTT-3') (Chen et al., 2004). A single amplicon of 1200 bp was obtained and sequenced (AgriGenome Labs Pvt. Ltd., India). BLAST searches showed 99 to $100 \%$ identity with Klebsiella variicola (GenBank Accession Nos. KF036184, MF370894, KX036863 and MF185379). Representative sequences of $K$. variicola from India were deposited in GenBank (MF179616, MF183971, MF183969, MF183973,
MF183974, MF183970, MF183972, MF373424 and MF370068). Cultures have been deposited in NBAIM (National Bureau of Agriculturally Important Microorganisms), Uttar Pradesh, India, with accession numbers: TB-2579, TB-2580, TB-2581, TB-2582, TB-2584, TB-2585, TB-2586 and TB-2587.

Klebsiella variicola was originally identified as a benign endosymbiont in plants but has since been associated with disease in humans and cattle (Rosenblueth et al., 2004). Fan et al. (2016) reported the occurrence of $K$. variicola causing banana soft rot in China. This is the first report of $K$. variicola causing soft rot on carrot in India.

\section{References}

Akbar A, Ahmad M, Azra, Neelam, Khan SZ, Ahmad Z, 2015.

Characterization of the causal organism of soft rot of tomatoes and other vegetables and evaluation of its most aggressive isolates. American Journal of Plant Sciences 6, 511-517. http://dx.doi.org/10.4236/ajps.2015.64055

Brenner DJ, Krieg NR, Staley JT, Garrity GM, eds, 2004. Bergey's Manual of Systematic Bacteriology. Volume Two: The Proteobacteria. New York, USA: Springer.

Chen L, Cai Y, Zhou G, Shi X, Su J, Chen G, Lin K, 2014. Rapid Sanger sequencing of the 16S rRNA gene for identification of some common pathogens. PLOS ONE 9. http://dx.doi.org/10.1371/journal.pone.0088886 Fan HC, Zeng L, Yang PW, Guo ZX, Bai TT, 2016. First report of banana soft rot caused by Klebsiella variicola in China. Plant Disease 100, 7-8. http://dx.doi.org/10.1094/PDIS-05-15-0586-PDN

Michalik B, Simon PW, Gabelman WH, 1992. Assessing susceptibility of carrot roots to bacterial soft rot. HortScience 27, 1020-1022.

Rosenblueth M, Martinez L. Silva J, Martínez-Romero E, 2004. Klebsiella variicola, a novel species with clinical and plant-associated isolates. Systematic and Applied Microbiology 27, 27-35. http://dx.doi.org/10.1078/0723-2020-00261

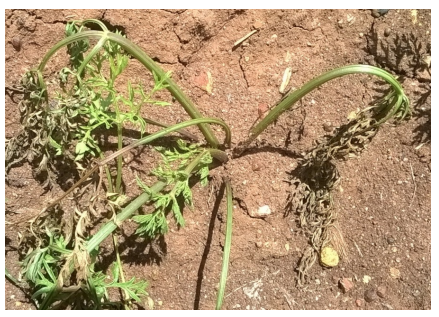

Figure 1

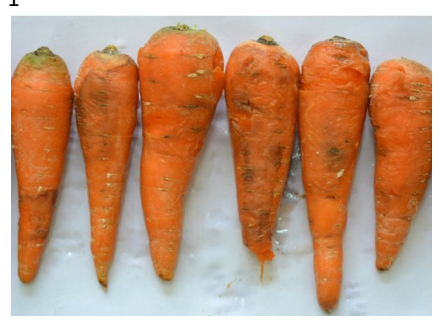

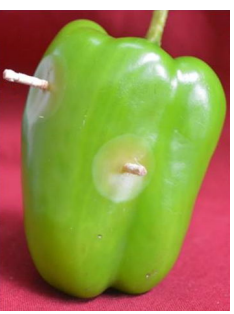

Figure 2

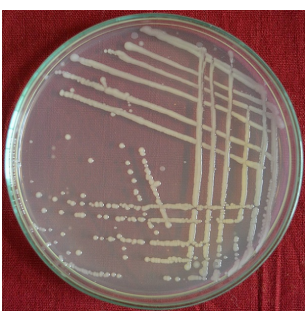

Figure 3

Figure 4

To cite this report: Chandrashekar BS, Prasannakumar MK, Puneeth ME, Teli K, Priyanka K, Mahesh HB, Desai RU, 2018. First report of bacterial soft rot of carrot caused by Klebsiella variicola in India. New Disease Reports 37, 21.

http://dx.doi.org/10.5197/j.2044-0588.2018.037.021

(c) 2018 The Authors

This report was published on-line at www.ndrs.org.uk where high quality versions of the figures can be found.

New Disease Reports is a peer-reviewed on-line journal published by the British Society for Plant Pathology,

for more information visit http://www.ndrs.org.uk/ 